\title{
L'« arrachement osseux » dorsal du carpe, une véritable fracture du triquetrum
}

\author{
Dorsal « bone avulsion » of the carpus, a true fracture of the triquetrum
}

\section{Tourdias · G. Tourdias}

Reçu le 4 avril 2013; accepté le 31 mai 2013

(C) SFMU et Springer-Verlag France 2013

Une patiente de 58 ans se présente aux urgences pour un poignet gauche douloureux suite à une chute de vélo. Initialement, seul le cliché de profil révèle une petite avulsion osseuse de la face postérieure du carpe en faveur d'une fracture triquétrale (Fig. 1A). Le scanner, réalisé secondairement après immobilisation, objective une véritable fracture non déplacée au niveau de la tubérosité postérieure du triquetrum (Fig. 1B). Cette lésion représente la forme la plus courante de la fracture du triquetrum qui constitue elle-même la deuxième fracture du carpe après celle du scaphoïde en terme de fréquence [1]. Cette fracture passe souvent inaperçue, car elle n'est visible sur les clichés standard du poignet que sur l'incidence de profil sous l'aspect d'un fragment osseux se projetant à la face dorsale du carpe [1]. La radiographie de face n'est pas contributive dans cette lésion du fait de la superposition du triquetrum avec le pisiforme. Cette atteinte du carpe est donc difficile à identifier et il ne faut notamment pas la confondre avec une atteinte de la corne postérieure du lunatum. Si le diagnostic précoce de cette fracture est essentiel pour une prise en charge thérapeutique adaptée, il repose sur une analyse rigoureuse du cliché de profil strict du poignet parfois aidé d'une imagerie en coupe.

\footnotetext{
D. Tourdias $(\bowtie)$
}

Service d'accueil des urgences - Smur,

centre hospitalier Sud Gironde, rue Paul Langevin,

F-33210 Langon, France

e-mail : tourdiasdamien@yahoo.fr

\section{G. Tourdias}

Service de chirurgie orthopédique et traumatologique, centre hospitalier Sud Gironde, rue Paul Langevin,

F-33210 Langon, France
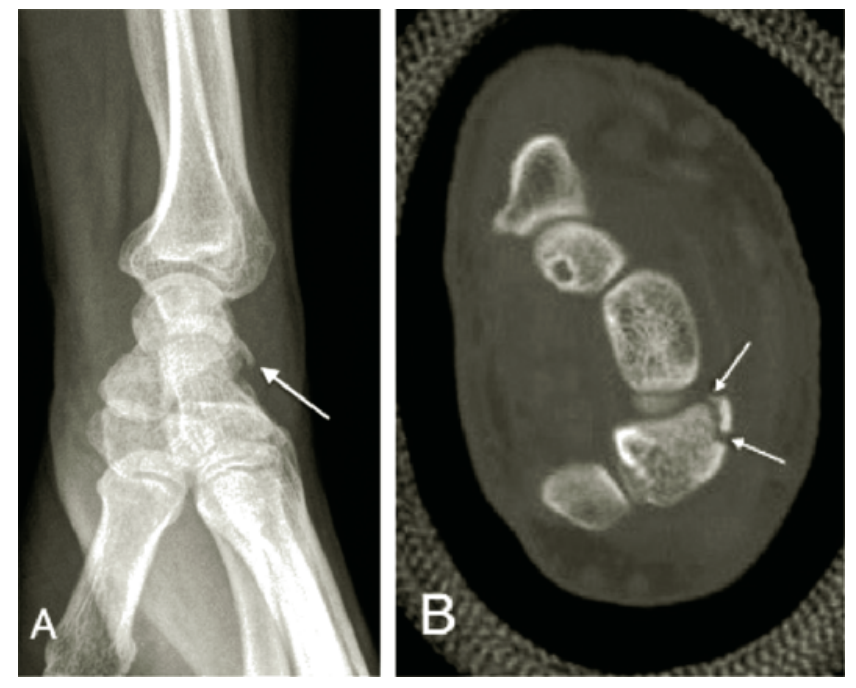

Fig. 1 Fracture du tubercule postérieur du triquetrum. A. Aspect faussement bénin d'《 arrachement osseux » postérieur du carpe. B. Coupe tomodensitométrique transversale du poignet confirmant la fracture

\section{Référence}

1. Höcker K, Menschik A (1994) Chip fractures of the triquetrum. Mechanism, classification and results. J Hand Surg Br 19:584-8 